CLINICAL STUDY

\title{
Polymorphisms in the adiponutrin gene are associated with increased insulin secretion and obesity
}

\author{
Lovisa E Johansson $^{1,4}$, Ulf Lindblad ${ }^{2,3,4}$, Charlotte A Larsson ${ }^{2,4}$, Lennart Råstam ${ }^{2,4}$ and Martin Ridderstråle ${ }^{1,4}$ \\ Departments of ${ }^{1}$ Clinical Sciences Malmö, Clinical Obesity and ${ }^{2}$ Community Medicine, Malmö University Hospital MAS, Lund University, Malmö, Sweden \\ S-20502, ${ }^{3}$ Skaraborg Institute, Skövde, Sweden S-54130 and ${ }^{4}$ Lund University Diabetes Centre (LUDC), Malmö, Sweden S-20502 \\ (Correspondence should be addressed to LE Johansson; Email: lovisa.johansson@med.lu.se)
}

\begin{abstract}
Objective: The insulin responsive adiponutrin or patatin-like phospholipase 3 (PNPLA3, previously $A D P N)$ gene shows association with obesity and in vitro adipocyte lipolysis. This study aimed to replicate the association between PNPLA3 variants and obesity, and to investigate their effect on insulin resistance and $\beta$-cell function.

Methods: rs738409 (Met148Ile) and rs2072907 (C to G) were genotyped using TaqMan allelic discrimination assay in a Swedish population-based sample $(n=1811)$. Oral glucose tolerance test (OGTT) with data from three time points $(0,30$, and $120 \mathrm{~min})$ were available from individuals under the age of 50 years $(n=973)$.

Results: Both variant alleles were associated with decreased prevalence of obesity $(P<0.05)$; odds ratio $0.75(0.61-0.93)$ per carried Ile-allele for rs738409 and 0.80 (0.64-1.00) per carried G-allele for rs2072907. For obesity as a quantitative trait, there was no association in the whole population, but in obese subjects body mass index (BMI; $P=0.023)$ and waist $(P=0.0098)$ were higher in carriers of the Ile-allele. The Ile-carriers also displayed decreased insulin secretion in response to OGTT (30 min insulin; $P=0.007$, insulinogenic index; $P=0.0051$ ) with no significant differences in fasting plasma glucose $(P=0.31)$, $\beta$-cell function (disposition index; $P=0.17)$ or homeostasis model of assessment insulin resistance (HOMA-IR; $P=0.063$ ). The correlation between BMI and HOMA-IR differed (Met/X versus Ile/Ile, $P=0.028$ ), Met-allele carriers were seemingly more insulin resistant at a lower BMI. The rs2072907 variant shows similar results for insulin secretion. The significance of this finding remained after adjusting for age, gender, and level of self-reported leisure-time physical activity. Conclusion: We confirm the association between PNPLA3 and obesity. In addition, the rs738409 variant was associated with insulin secretion. There seems to be a differential effect of the Ile-allele depending on the degree of obesity, possibly as a consequence of insulin resistance.
\end{abstract}

European Journal of Endocrinology 159 577-583

\section{Introduction}

Adiponutrin (PNPLA3, previously ADPN) is a recently described adipocyte protein, which seems to harbor both lipolytic and lipogenic properties (1-5). Insulin regulates the gene positively in a glucose-dependent fashion. As a consequence of the elevated fasting insulin levels associated with obesity, PNPLA3 mRNA was increased in obese compared with lean human subjects in our previous study (6) where it otherwise would be greatly reduced in the fasting state (7-9). Subjects that are insulin resistant have a lesser increase of adipose tissue PNPLA 3 mRNA expression despite elevated insulin levels (6). It is currently unknown whether this inability to increase PNPLA3 is of pathophysiological importance.

In a recent study by us, we have shown that the wildtype alleles of common variants in the PNPLA3 gene were associated with human obesity in Swedish case-control material $\left(n_{\text {case/control }}=234 / 234\right)(6)$. The risk allele of rs2072907 was also associated with decreased subcutaneous but not visceral adipose tissue PNPLA3 mRNA expression (6). Since adipocytes from carriers of this allele exhibited increased basal lipolysis it was suggested that the main function of adiponutrin may be lipogenic rather than lipolytic, i.e., that decreased expression would result in a net increase in lipolysis due to loss of lipogenesis.

The aim of this study was to replicate previous findings of an association between PNPLA3 variants and obesity and to investigate whether there is an effect of these genetic variants on insulin sensitivity and secretion as forerunners of obesity-associated development of diabetes.

\section{Subjects and methods}

\section{Subjects}

This investigation is based on data from a populationbased study in Vara, a small community in a rural area 
of southwestern Sweden, as part of the Skaraborg Project $(10,11)$. A total of 1811 subjects were surveyed (Table 1). Obesity was defined as a body mass index (BMI) $\geq 30 \mathrm{~kg} / \mathrm{m}^{2}$. A diagnosis of diabetes was confirmed if there was a history of a physician's diagnosis, and in new cases when the fasting plasma glucose value was $\geq 7.0 \mathrm{mmol} / \mathrm{l}$ (twice) and/or when the $2 \mathrm{~h}$ plasma glucose value from an oral glucose tolerance test (OGTT) was $\geq 11.1 \mathrm{mmol} / \mathrm{l}$. Self-reported leisure-time physical activity (LTPA) was divided into four different categories, ranging from sedentary to strenuous exercise $(11,12)$. Per definition low level of LTPA includes subjects with an estimated LTPA of 1 and 2 and high level of LTPA subjects with LTPA of 3 and 4. All subjects underwent an OGTT (75 g glucose) with blood drawn at 0 and $120 \mathrm{~min}$. Blood drawn at $30 \mathrm{~min}$ were available for all subjects under the age of 50 years and these data were used for further analysis $(n=973$, non-diabetic subjects, age 40 (36-45) years, BMI 25.4 $(23.1-28.1) \mathrm{kg} / \mathrm{m}^{2}$ and $54 \%$ males). Serum insulin was analyzed using ELISA with $<0.3$ percent crossreactivity for pro-insulin (DRACO Diagnostics Ltd, Ely, Cambridgeshire, UK) (13). Data are presented as pmol/l but calculations using insulin values are based on $\mu \mathrm{U} / \mathrm{ml}$. Plasma glucose levels were analyzed using standard procedures. Insulin resistance was estimated by homeostasis model of assessment insulin resistance (HOMA-IR), calculated by dividing the product of fasting glucose $(\mathrm{mmol} / \mathrm{l})$ and insulin levels $(\mu \mathrm{U} / \mathrm{ml})$ by 22.5 (14). Insulinogenic index ((insulin at $30 \mathrm{~min}-$ insulin at baseline)/glucose at $30 \mathrm{~min}$ ), disposition index (insulinogenic index/HOMA-IR) and area under the curve (AUC) were calculated. The regional ethical review board of Göteborg University approved the study and informed written consent was obtained from the subjects prior to their participation.

\section{Genotyping}

DNA was extracted from whole blood by using Qiagen MiniPrep at the DNA/RNA Genotyping Lab, SWEGENE Resource Center for Profiling Polygenic Disease, Lund University, Malmö University Hospital, Malmö, Sweden. Two single nucleotide polymorphisms (SNPs) were chosen for genotyping based on a previous study (6). Two SNPs showed strong association with obesity, the rs1010022 and rs2072907. These two SNPs were in high linkage disequilibrium and therefore only the defined tagSNP rs2072907 was chosen for genotyping in this study. SNP rs738409 was chosen because it results in an amino acid switch (Met148Ile) in the important lipolytic and lipogenic patatin domain in the protein and has previously shown marginal association with obesity (6). Genotyping was performed using TaqMan allelic discrimination on the ABI 7900HT from Applied Biosystems (Foster City, CA, USA, Assay by Design). Sequences for rs2072907 forward: CTGGAAGGCAGGTGTAACCA; reverse: CTTTGTGGGCTCCATCTACATATCT; probes: CAGTTGTTATAAACGAACACTA (VIC) and CAGTTGTTATAAACCAACACTA (FAM). Sequences for rs738409 forward: GCTTTCACAGGCCTTGGTATG; reverse: GGAGGGATAAGGCCACTGTAGAA; probes: VIC-TTCCTGCTTCATCCC-MGB and FAM-TTCCTGCTTCATGCC-MGB. Genotyping was performed in $5 \mu \mathrm{l}$ reaction volumes with $5 \mathrm{ng}$ DNA and Universal TaqMan $2 \times$ PCR MasterMix according to the manufacturer's recommendations (Applied Biosystems). Success rate for all genotyping was $>99 \%$. For both SNPs, genotyping failed in six subjects. Using the same method, $3-5 \%$ of the samples were rerun with a concordance of $100 \%$. There were no deviations from Hardy-Weinberg equilibrium.

\section{Statistical analysis}

The $\chi^{2}$-test was used to compare the proportions and frequencies. Associations between obesity and categorical variables were analyzed by using binary logistic regression in SPSS v15.0 (Chicago, IL, USA) defining the change in odds ratio (OR) per allele, expressed as OR with 95\% confidence interval. Analysis of covariance was used to investigate the effect of genotypes on clinical variables. Age, gender, and LTPA were used as covariates. Regression was analyzed using Pearson's correlation in subgroups based on a dominant model of rs738409 (Met/X versus Ile/Ile) defined by data from Table 3. The slopes of the two regression lines were compared using seemingly unrelated estimation

Table 1 Characteristics of the study population.

\begin{tabular}{llll}
\hline & All & Male & Female \\
\hline Age (years) & $46(38-56) n=1811$ & $46(38-56) n=904$ & $46(38-56) n=907$ \\
BMI (kg/m $\left.{ }^{2}\right)$ & $26.0(23.7-29.3) n=1809$ & $26.3(24.4-29.0) n=904$ & $25.5(23.1-29.6) n=905$ \\
fP-Insulin (pmol/l) & $30.5(20.3-46.7) n=1800$ & $32.0(21.5-49.3) n=900$ & $28.6(19.1-44.0) n=900$ \\
fP-Glucose (mmol/l) & $5.2(4.9-5.6) n=1811$ & $5.3(5.0-5.7) n=904$ & $5.1(4.8-5.4) n=907$ \\
HOMA-IR & $1.16(0.76-1.90) n=1800$ & $1.29(0.82-2.06) n=900$ & $1.08(0.72-1.74) n=900$ \\
Diabetes (\%) & $6.0 n=108$ & $6.3 n=57$ & $5.6 n=51$ \\
BMI $\geq 30(\%)$ & $20.9 n=379$ & $19.0 n=172$ & $22.8 n=207$ \\
Low LTPA (\%) & $65.6 n=1188$ & $61.2 n=553$ & $70.0 n=635$ \\
\hline
\end{tabular}

Data are presented as median with interquartile range within brackets (25th-75th); $n$, number of observations. For diabetes status, BMI and LTPA data are presented as \%. HOMA-IR, homeostasis model of assessment (14); LTPA, leisure-time physical activity. 
test in STATA. $P<0.05$ was considered statistically significant. Data were log transformed for normal distribution and presented as median with interquartile range (25th-75th percentile) within brackets or mean \pm s.e.m. Statistical calculations were performed using Number Cruncher Statistical Systems 2000 software (NCSS, Kaysville, UT, USA) unless stated otherwise.

With regards to the risk allele frequencies from the previous study $(73.3 \%$ in controls and $78.8 \%$ in cases of the rs738409 Met allele and 75.9\% in controls and $83.5 \%$ in cases of the rs 2072907 C-allele), when $\alpha$ was set at 0.05 and the current sample size of 379 cases and 1432 controls were used the power for SNP rs738409 would be 59 and $90 \%$ for rs 2072907 .

\section{Results}

\section{Description of population}

The study population has been described previously (11). In brief, the distribution of males and females was equal and showed no differences in age (Table 1). The prevalence of diabetes was $6.0 \%$. Males had slightly higher BMI and reported higher LTPA compared with females. There was an increased prevalence of obesity among subjects reporting low LTPA (OR 2.27 (1.713.01), $P<0.0001)$. BMI was $26.5(24.1-30.0)$ and $25.2(23.3-27.7) \mathrm{kg} / \mathrm{m}^{2}$ for subjects reporting low and high LTPA respectively $(P<0.0001)$.

The minor allele frequencies in the whole population were 21 and $17 \%$ for rs738409 and rs2072907 respectively. There were no differences in age or gender between rs738409 genotype carriers but there was a difference in age concerning rs2072907 $(P=0.0067$, Table 2).

\section{Genetic variants in the adiponutrin gene show association with obesity}

Binary logistic regression suggested a decreased risk of obesity with an OR of $0.75(0.61-0.93)$ per Ile-allele of the rs738409 polymorphism when adjusting for age, gender, and LTPA, $P=0.009$ (adjusting for age and gender only, OR $0.77(0.63-0.95)$ per Ile-allele, $P=0.016)$. Similarly, for rs2072907, there was a decreased risk of obesity with an OR of 0.80 (0.641.00) per carried G-allele adjusted for age, gender, and LTPA, $P=0.049$ (adjusting for age and gender only, OR $0.82(0.66-1.02)$ per carried G-allele, $P=0.069)$.

There was no significant difference when analyzing obesity as a quantitative trait in the whole population for either polymorphism (Table 2). However, analyzing the traits in obese subjects separately revealed a significant difference. BMI was higher in carriers of the rs738409 Ileallele after adjustments for age, gender, and LTPA (Met/Met 32.2 (31.1-34.9) $n=255$, Met/Ile 33.2 (31.5-37.1) $n=114$ and Ile/Ile 32.2 (31.0-38.2) $\left.\mathrm{kg} / \mathrm{m}^{2} n=9, P=0.023\right)$. The significance of this difference disappeared when adjusting for HOMA-IR $(P=0.12)$. Waist was also associated with the rs738409 genotypes after adjustments for age, gender, and LTPA (Met/Met 105 (98-111) $n=254$, Met/Ile $107(100-115) n=114$ and Ile/Ile $109(106-116) \mathrm{cm} n=9, P=0.0098)$ but not when adjusting for HOMA-IR $(P=0.050)$. There were no effects of the rs2072907 polymorphism on quantitative obesity traits in the obese (data not shown).

\section{Genetic variants in the adiponutrin gene are associated with insulin secretion and insulin resistance}

Data on insulin levels at 0,30 , and $120 \mathrm{~min}$ from an OGTT were available from 973 non-diabetic individuals, all of whom were under the age of 50 . With no

Table 2 Differences in clinical characteristics concerning two genetic variants in the adiponutrin (PNPLA3) gene.

\begin{tabular}{|c|c|c|c|c|c|}
\hline rs738409 & Met/Met & Met/lle & Ile/lle & $P$ & $\boldsymbol{P}_{\text {adj }}$ \\
\hline $\operatorname{Sex}(m / f)$ & $585 / 548$ & $275 / 316$ & $42 / 37$ & 0.11 & - \\
\hline Age (years) & $46(39-57) n=1133$ & $45(37-54) n=591$ & $45(38-55) n=79$ & 0.14 & - \\
\hline $\mathrm{BMI}\left(\mathrm{kg} / \mathrm{m}^{2}\right)$ & $26.2(23.6-29.6) n=1133$ & $25.8(23.8-29.0) n=591$ & $26.0(23.8-28.0) n=79$ & 0.59 & 0.71 \\
\hline Weight (kg) & $78.7(69.0-89.5) n=1133$ & $77.6(68.4-88.8) n=591$ & $79.2(68.3-87.8) n=79$ & 0.58 & 0.79 \\
\hline Waist (cm) & $90(81-99) n=1129$ & $89(80-97) n=591$ & $89(81-97) n=78$ & 0.36 & 0.64 \\
\hline HOMA-IR & $1.16(0.76-1.89) n=1128$ & $1.18(0.77-1.92) n=588$ & $1.01(0.61-1.77) n=78$ & 0.16 & 0.16 \\
\hline rs2072907 & CC & CG & GG & $P$ & $\boldsymbol{P}_{\text {adj }}$ \\
\hline $\operatorname{Sex}(m / f)$ & $628 / 612$ & $242 / 257$ & $32 / 32$ & 0.72 & - \\
\hline Age (years) & $46(39-57) n=1240$ & $45(37-53) n=499$ & $48(38-58) n=64$ & 0.0067 & - \\
\hline BMI $\left(\mathrm{kg} / \mathrm{m}^{2}\right)$ & $26.2(23.8-29.4) n=1240$ & $25.8(23.6-29.0) n=499$ & $25.9(23.6-28.6) n=64$ & 0.25 & 0.37 \\
\hline Weight (kg) & $78.9(69.2-89.5) n=1240$ & $77.5(67.8-88.8) n=499$ & $77.8(67.6-87.9) n=64$ & 0.30 & 0.55 \\
\hline Waist (cm) & $90(81-99) n=1237$ & $88(80-97) n=498$ & $88(80-99) n=63$ & 0.18 & 0.75 \\
\hline HOMA-IR & $1.17(0.76-1.92) n=1234$ & $1.17(0.77-1.86) n=497$ & $1.03(0.63-1.99) n=63$ & 0.48 & 0.56 \\
\hline
\end{tabular}

$P$ values were calculated using Kruskal-Wallis. Adjusted $P$ values using GLM-ANOVA with age and sex as covariates. The $P$ values for genotype frequencies were calculated using $\chi^{2}$-test. Data are presented as median with interquartile range within brackets (25th-75th); $n$, number of observations; HOMA-IR, homeostasis model of assessment (14). 
Table 3 The effect of single nucleotide polymorphism (SNP) rs738409 and rs2072907 in the adiponutrin (PNPLA3) gene on measures of insulin secretion during an oral glucose tolerance test (OGTT) and on insulin resistance in 973 non-diabetic individuals.

\begin{tabular}{|c|c|c|c|c|c|c|c|c|}
\hline & \multicolumn{4}{|c|}{ rs738409 } & \multicolumn{4}{|c|}{ rs2072907 } \\
\hline & Met/Met $n=597$ & Met/lle $n=329$ & Ile/lle $n=47$ & $P$ value & CC $n=647$ & CG $n=291$ & GG $n=35$ & $P$ value \\
\hline Age (years) & $41(36-45)$ & $40(36-45)$ & $40(36-45)$ & 0.32 & $41(36-45)$ & $40(35-45)$ & $41(36-46)$ & 0.062 \\
\hline BMI $\left(\mathrm{kg} / \mathrm{m}^{2}\right)$ & $25.2(23.0-28.0)$ & $25.4(23.2-28.1)$ & $25.4(23.6-27.6)$ & 0.44 & $25.3(23.1-28.1)$ & $25.3(23.1-27.7)$ & $25.9(23.4-28.9)$ & 0.62 \\
\hline $\begin{array}{l}\text { fP-Glucose } 0 \mathrm{~min} \\
(\mathrm{mmol} / \mathrm{l})\end{array}$ & $5.1(4.8-5.4)$ & $5.1(4.9-5.4)$ & $5.1(4.8-5.5)$ & 0.31 & $5.1(4.8-5.4)$ & $5.1(4.8-5.4)$ & $5.2(4.8-5.6)$ & 0.20 \\
\hline $\begin{array}{l}\text { P-Glucose } 30 \mathrm{~min} \\
(\mathrm{mmol} / \mathrm{l})\end{array}$ & $7.4(6.4-8.3)$ & $7.2(6.2-8.3)$ & $7.2(5.9-8.6)$ & 0.50 & $7.3(6.4-8.3)$ & $7.2(6.2-8.3)$ & $7.4(6.1-9.0)$ & 0.60 \\
\hline $\begin{array}{l}\text { P-Glucose } 120 \mathrm{~min} \\
(\mathrm{mmol} / \mathrm{l})\end{array}$ & $4.9(4.1-5.6)$ & $5.0(4.1-5.9)$ & $4.7(3.8-6.3)$ & 0.87 & $4.9(4.1-5.7)$ & $4.9(4.0-5.9)$ & $5.3(4.1-6.1)$ & 0.92 \\
\hline $\begin{array}{l}\text { fP-Insulin } 0 \text { min } \\
(\mathrm{pmol} / \mathrm{l})\end{array}$ & $28.1(20.0-41.0)$ & $30.2(20.8-43.1)$ & $25.8(16.4-35.3)$ & 0.064 & $28.3(20.0-41.8)$ & $28.9(20.0-42.0)$ & $29.1(18.4-43.1)$ & 0.63 \\
\hline $\begin{array}{l}\text { P-Insulin } 30 \mathrm{~min} \\
\text { (pmol//) }\end{array}$ & $225.6(159.7-330.8)$ & $238.2(162.0-337.1)$ & $189.1(124.9-286.4)$ & $0.0070^{\mathrm{a}}$ & $224.3(160.6-323.6)$ & $238.1(156.8-340.7)$ & $213.7(134.8-290.1)$ & $0.021^{\mathrm{a}}$ \\
\hline $\begin{array}{l}\text { P-Insulin } 120 \mathrm{~min} \\
\text { (pmol/l) }\end{array}$ & $132.2(86.8-200.8)$ & $142.9(97.6-216.2)$ & $142.3(81.6-224.1)$ & $0.50^{\mathrm{a}}$ & $133.8(87.6-205.7)$ & $139.4(92.7-215.4)$ & $148.4(100.0-211.0)$ & $0.93^{\mathrm{a}}$ \\
\hline AUC (insulin) & 3414 (2437-4904) & 3624 (2537-4952) & $2854(2080-4760)$ & 0.050 & 3435 (2486-4897) & 3582 (2478-4973) & 3477 (2168-4697) & 0.23 \\
\hline $\begin{array}{l}\text { First-phase } A \cup C^{b} \\
\text { (insulin) }\end{array}$ & $641(461-928)$ & $680(461-930)$ & $528(356-793)$ & 0.0019 & $641(464-911)$ & $671(443-944)$ & $587(375-798)$ & 0.032 \\
\hline HOMA-IR & $1.06(0.74-1.62)$ & $1.13(0.77-1.65)$ & $0.98(0.61-1.54)$ & 0.063 & $1.07(0.74-1.62)$ & $1.10(0.76-1.63)$ & $1.05(0.71-1.85)$ & 0.54 \\
\hline Insulinogenic index ${ }^{c}$ & $4.5(3.0-6.8)$ & $4.6(3.3-6.9)$ & $4.1(2.6-6.0)$ & 0.0051 & $4.5(3.0-6.7)$ & $4.7(3.5-6.9)$ & $4.2(2.4-6.0)$ & 0.011 \\
\hline Disposition index ${ }^{d}$ & $4.2(2.7-6.5)$ & $4.1(2.5-6.2)$ & $3.9(2.6-6.0)$ & 0.17 & $4.1(2.6-6.5)$ & $4.3(2.8-6.6)$ & $3.9(2.1-5.8)$ & 0.13 \\
\hline
\end{tabular}

Data are presented as median with interquartile range within brackets (25th-75th). $P$ values are calculated using ANCOVA including age, gender, BMI, physical activity. AUC, area under the curve; HOMA-IR, homeostasis model of assessment (14).

Insulin at baseline in the model.

${ }^{\mathrm{b}}$ First-phase AUC, AUC between 0 and $30 \mathrm{~min}$

(Insulin at $30 \mathrm{~min}$-insulin at baseline)/glucose at $30 \mathrm{~min}$.

Insulinogenic index/HOMA-IR. 
differences in plasma glucose at these time points, firstphase insulin secretion defined as insulin at $30 \mathrm{~min}$ during OGTT was significantly different between Met148Ile genotypes (Table 3). The same relationship was found for AUC between 0 and 30 min (Table 3 and Fig. 1). Insulinogenic index was higher in carriers of the Met-allele but insulin resistance measured as HOMA-IR, and $\beta$-cell function measured as disposition index, did not differ significantly (Table 3). Moreover, the slope of the regression line when looking at the correlation between BMI and HOMA-IR was significantly steeper in homozygous carriers of the variant Ile-allele than in carriers of the Met-allele $(R=0.67$ vs $0.5367, P=0.028$, Fig. 2) suggesting that the carriers of the Met-allele are more insulin resistant at a lower BMI. There were no differences between the correlations for disposition index $(R=-0.31$ vs -0.16 , $P=0.61$, Fig. 2). Results concerning rs2072907
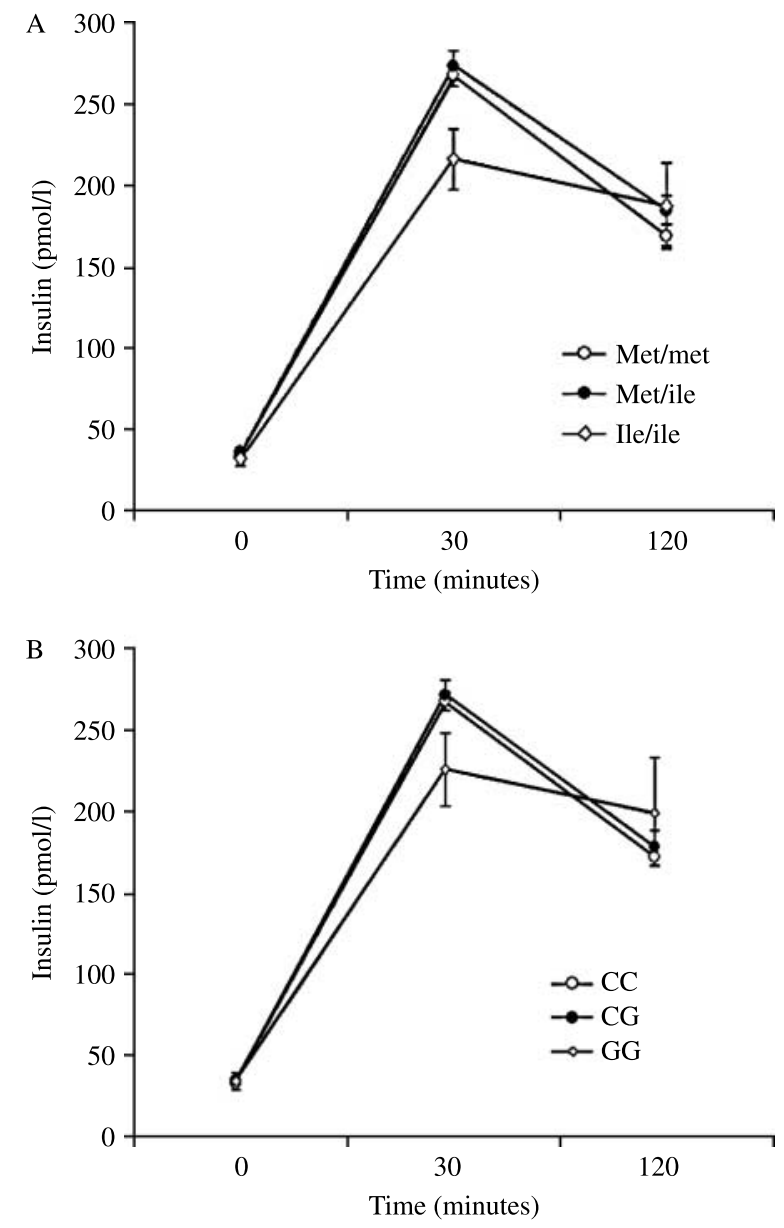

Figure 1 The effect of genetic variants in the adiponutrin gene on insulin levels. Plasma insulin levels measured during an oral glucose tolerance test (OGTT) in non-diabetic individuals under the age of 50 in a Swedish population divided on the genetic variants $(A)$ rs738409 and (B) rs2072907 in the adiponutrin (PNPLA3) gene. Data are presented as mean \pm S.E.M. trended towards the same differences (Table 3). Adjusting the analysis regarding rs738409 for SNP rs2072907 did not change the results but performing the analysis vice versa attenuated the findings (data not shown).

\section{Discussion}

Variations in the adiponutrin gene have previously been associated with obesity, level of adipose tissue mRNA expression, and basal lipolysis (6). We sought to
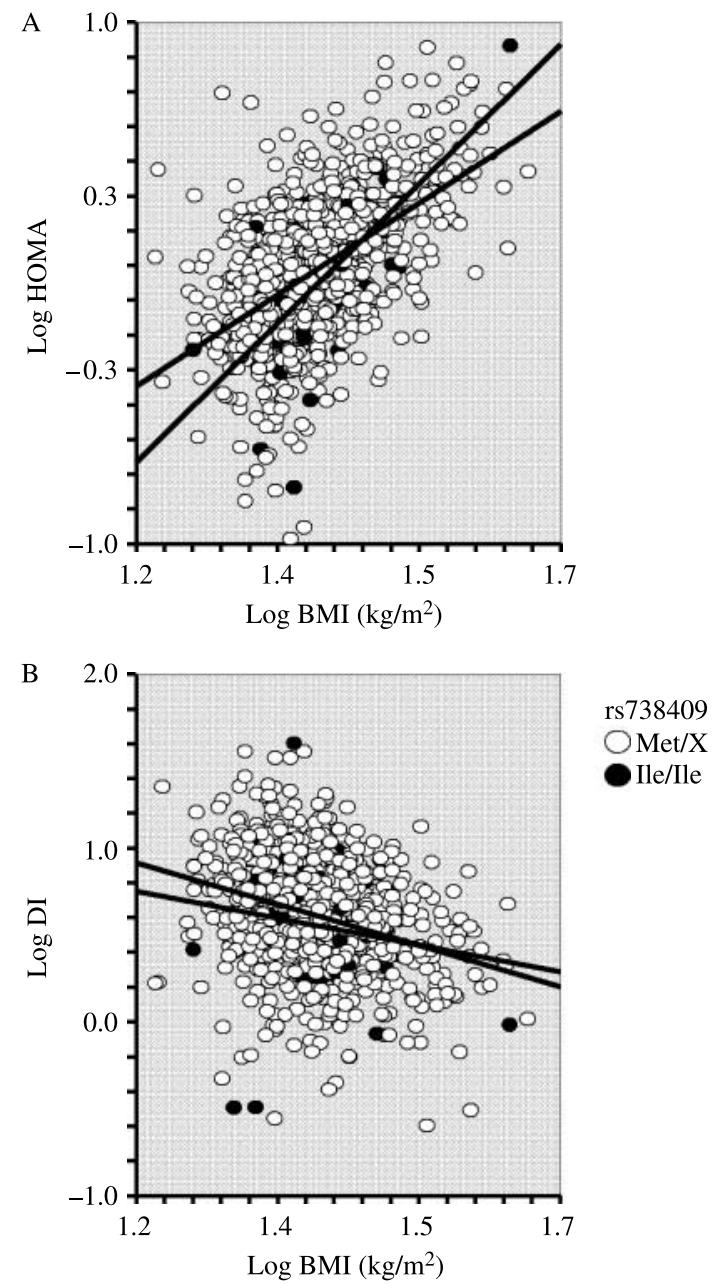

Figure 2 The effect of genetic variants in the adiponutrin gene on the correlation between obesity and insulin resistance. The correlation between $\mathrm{BMI}$ and $(\mathrm{A})$ insulin resistance defined by HOMA-IR and (B) $\beta$-cell function defined by disposition index (DI) from an oral glucose tolerance test (OGTT) in non-diabetic individuals under the age of 50 in a Swedish population divided on carriers of the genetic variant rs738409. Open circles and white lines represent carriers of Met/X ( $n=926)$ whereas filled circles and black lines represent homozygous carriers of the variant lle-allele $(n=47)$. (A) $R_{\text {Met } / \mathrm{X}}=0.53, R_{\text {Ile/lle }}=0.67$, and $P_{\text {difference }}=0.028$ and (B) $R_{\mathrm{Met} / \mathrm{X}}=-0.31, R_{\mathrm{lle} / \mathrm{le}}=-0.16$, and $P_{\text {difference }}=0.61$. Data are log transformed for normal distribution, correlation calculated using Pearson and $P$ values using the seemingly unrelated estimation test. 
replicate the obesity finding and further extend the study by investigating the effect of PNPLA3 on insulin sensitivity and secretion. For this purpose, two of the most interesting SNPs were selected from the original publication, a strongly associated tagSNP rs272907 and a coding variant in the patatin domain of the protein rs738409, to be genotyped in a Swedish population-based study. We found that the coding SNP rs738409 in the PNPLA3 gene that showed association with both obesity and measures of insulin secretion were wild-type Met-allele carriers having increased insulin secretion.

In a previous study we found that four PNPLA3 SNPs (rs738409, rs2076211, rs2072907, and rs1010022) were associated with obesity, two of them (the tagSNP rs2072907 and rs1010022) remaining significant after correction for multiple testing (6). Contrary to our previous investigation, rs2072907, a non-coding variant located in intron 5 , had only minor or no effects on the risk of obesity in the current study. Previous data suggested that the two SNPs were located in two separate haplotype blocks although some linkage was seen between the two $\left(D^{\prime}=0.87 \quad(0.82-0.92)\right.$, $\left.r^{2}=0.60\right)$. Here, the two SNPs show similar linkage disequilibrium $\left(D^{\prime}=0.90(0.86-0.92), r^{2}=0.65\right)(6)$. We cannot exclude that rs2072907 reflects the effect of another causative variant(s), possibly rs738409, by linkage disequilibrium in the previous study. It is important to bear in mind that there are differences in both design and sample selection between the studies. The subjects in this study were randomly selected from a population while the previous study was based on severely obese patients referred to an obesity clinic at the hospital (BMI 32.5 (31.1-35.7) vs 40.3 (35.5-45.3) $\mathrm{kg} / \mathrm{m}^{2}$ ). Differences in the level of physical activity might also influence the difference between the two studies. We lack these data for the previously investigated patient sample but due to their severe obesity the level of physical activity is expected to be limited.

In this Swedish population-based study we found that the wild-type Met-allele of rs738409 shows association with obesity and measures of increased insulin secretion. The fact that the Met-allele is associated with both increased risk of obesity and increased insulin secretion but not directly with insulin resistance in the whole population may at first seem contradictory. Focusing on the obese subjects separately, the variant Ile-allele rather than the previously defined risk, the Met-allele, was associated with a slightly higher BMI (6). A difference in insulin resistance might have explained these contradictory results but there does not seem to be an immediate effect of rs738409 on HOMA-IR. A possible key to this issue comes from the correlation between BMI and insulin resistance. This analysis revealed that insulin resistance rose more rapidly with increasing BMI in carriers of the Ile/Ile genotype compared with Met-allele carriers. In other words, carriers of the Ile-allele may be more sensitive to developing disease as a consequence of being obese. However, in light of the greater degree of insulin resistance at lower levels of BMI found in carriers of the Met-allele, obesity may have developed as an anabolic consequence of the resulting increase in insulin secretion in these subjects. In fact, a pilot study investigating rs738409 in a small type 2 diabetes case-control material suggests that the Ile/Ile genotype is more frequent in type 2 diabetes cases (data not shown). There are other examples of genetic variants that exhibit these apparently perplexing associations. For example, whereas the Ala-allele of the Pro12Ala polymorphism of the peroxisome proliferator-activated receptor gamma (PPARG) gene is associated with a protection against type 2 diabetes, it is also associated with an increased risk of developing obesity $(15,16)$. In this case, the common denominator is the increased insulin sensitivity associated with PPARG 12Ala. Both increased insulin secretion and enhanced insulin sensitivity will ultimately lead to the same result, a small but significant weight gain over time. Clearly, the effect of rs 738409 on both insulin resistance and type 2 diabetes will require further investigation.

Adipose tissue PNPLA3 mRNA expression is influenced by nutritional status, insulin and insulin sensitivity, degree of obesity, and genetic variants in the PNPLA3 gene $(3,4,6-9,17-19)$. The knowledge regarding the role of adiponutrin in the adipocyte or any other cell is still very limited, making it difficult to functionally understand a link between adiponutrin and insulin secretion. Likely, the link resides in insulin resistance rather than a direct interaction between the two in the insulin secreting $\beta$-cell. All the same, since fatty acids have been suggested to play a pivotal role both in the production and secretion of insulin $(20,21)$ and rs738409 is located in the part of the gene coding for the so-called patatin domain, which is responsible for catalyzing the cleavage of fatty acids from triglycerides (22), a direct link cannot be excluded. Hypothetically, the PNPLA3 protein could influence insulin secretion through either its lipolytic or lipogenic properties by altering the load of fatty acids into the circulation and/or in the $\beta$-cell itself.

In conclusion, we have presented data confirming the association between a genetic variant in the adiponutrin gene with obesity and further extend the findings by showing an association with insulin secretion in a Swedish population-based sample. Whether this association is a cause or a consequence of the association with obesity remains to be elucidated.

\section{Declaration of interest}

The authors declare that there is no conflict of interest that would prejudice the impartiality of this scientific work.

\section{Funding}

This study was supported by grants from the Swedish Research Council, the Skaraborg Institute, the Health and Medical Care Committee of 
the Regional Executive Board of the Region Västra Götaland, Skane Region, Lund University, and the following foundations: Novo Nordisk, Påhlsson, Craaford, Borgström, Bergvall, Zoegas, Hierta, and Swedish Diabetes Foundation.

\section{Acknowledgements}

We thank M Persson, S Andersson, M Nyholm, and A C Agardh for their excellent work with the data collection, and we are greatly indebted to the citizens of Vara for their participation in the study. Special thanks to Prof. Leif Groop for reading and commenting on this manuscript and to Peter Almgren for the help to solve statistical problem.

\section{References}

1 Baulande S, Lasnier F, Lucas M \& Pairault J. Adiponutrin, a transmembrane protein corresponding to a novel dietary- and obesity-linked mRNA specifically expressed in the adipose lineage. Journal of Biological Chemistry 2001276 33336-33344.

2 Jenkins CM, Mancuso DJ, Yan W, Sims HF, Gibson B \& Gross RW. Identification, cloning, expression, and purification of three novel human calcium-independent phospholipase A2 family members possessing triacylglycerol lipase and acylglycerol transacylase activities. Journal of Biological Chemistry 2004 279 48968-48975.

3 Kershaw EE, Hamm JK, Verhagen LA, Peroni O, Katic M \& Flier JS. Adipose triglyceride lipase: function, regulation by insulin, and comparison with adiponutrin. Diabetes 200655 148-157.

4 Lake AC, Sun Y, Li JL, Kim JE, Johnson JW, Li D, Revett T, Shih HH, Liu W, Paulsen JE \& Gimeno RE. Expression, regulation, and triglyceride hydrolase activity of adiponutrin family members. Journal of Lipid Research 200546 2477-2487.

5 Wilson PA, Gardner SD, Lambie NM, Commans SA \& Crowther DJ. Characterization of the human patatin-like phospholipase family. Journal of Lipid Research 200647 1940-1949.

6 Johansson LE, Hoffstedt J, Parikh H, Carlsson E, Wabitsch M, Bondesson AG, Hedenbro J, Tornqvist H, Groop L \& Ridderstråle M. Variation in the adiponutrin gene influences its expression and associates with obesity. Diabetes 200655 826-833.

7 Liu YM, Moldes M, Bastard JP, Bruckert E, Viguerie N, Hainque B, Basdevant A, Langin D, Pairault J \& Clément K. Adiponutrin: a new gene regulated by energy balance in human adipose tissue. Journal of Clinical Endocrinology and Metabolism 200489 2684-2689.

8 Moldes M, Beauregard G, Faraj M, Peretti N, Ducluzeau PH, Laville M, Rabasa-Lhoret R, Vidal H \& Clément K. Adiponutrin gene is regulated by insulin and glucose in human adipose tissue. European Journal of Endocrinology 2006155 461-468.

9 Faraj M, Beauregard G, Loizon E, Moldes M, Clément K, Tahiri Y, Cianflone K, Vidal H \& Rabasa-Lhoret R. Insulin regulation of gene expression and concentrations of white adipose tissue-derived proteins in vivo in healthy men: relation to adiponutrin. Journal of Endocrinology $2006191427-435$.
10 Nyholm M, Gullberg B, Rastam L \& Lindblad U. Higher education and more physical activity limit the development of obesity in a Swedish rural population. The Skaraborg Project. International Journal of Obesity 200832 533-540.

11 Ridderstråle M, Johansson LE, Rastam L \& Lindblad U. Increased risk of obesity associated with the variant allele of the PPARGC1A Gly482Ser polymorphism in physically inactive elderly men. Diabetologia $200649496-500$.

12 Nyholm M, Gullberg B, Merlo J, Lundqvist-Persson C, Råstam L \& Lindblad U. The validity of obesity based on self-reported weight and height: implications for population studies. Obesity 200715 197-208.

13 Andersen L, Dinesen B, Jorgensen PN, Poulsen F \& Roder ME. Enzyme immunoassay for intact human insulin in serum or plasma. Clinical Chemistry 199339 578-582.

14 Matthews DR, Hosker JP, Rudenski AS, Naylor BA, Treacher DF \& Turner RC. Homeostasis model assessment: insulin resistance and beta-cell function from fasting plasma glucose and insulin concentrations in man. Diabetologia 1985128 412-419.

15 Altshuler D, Hirschhorn JN, Klannemark M, Lindgren CM, Vohl MC, Nemesh J, Lane CR, Schaffner SF, Bolk S, Brewer C, Tuomi T, Gaudet D, Hudson TJ, Daly M, Groop L \& Lander ES. The common PPAR $\gamma$ Pro12Ala polymorphism is associated with decreased risk of type 2 diabetes. Nature Genetics 200026 76-80.

16 Masud S \& Ye S. The SAS Group. Effect of the peroxisome proliferator activated receptor-gamma gene Pro12Ala variant on body mass index: a meta-analysis. Journal of Medical Genetics 2003 40 773-780.

17 Bertile F \& Raclot T. Differences in mRNA expression of adipocytederived factors in response to fasting, refeeding and leptin. Biochimica et Biophysica Acta 20041683 101-109.

18 Polson DA \& Thompson MP. Adiponutrin mRNA expression in white adipose tissue is rapidly induced by meal-feeding a highsucrose diet. Biochemical and Biophysical Research Communications $2003301261-266$.

19 Polson DA \& Thompson MP. Macronutrient composition of the diet differentially affects leptin and adiponutrin mRNA expression in response to meal feeding. Journal of Nutritional Biochemistry 2004 $15242-246$.

20 Nolan CJ, Madiraju MS, Delghingaro-Augusto V, Peyot ML \& Prentki M. Fatty acid signaling in the beta-cell and insulin secretion. Diabetes 200655 S16-S23.

21 Yaney GC \& Corkey BE. Fatty acid metabolism and insulin secretion in pancreatic beta cells. Diabetologia 200346 1297-1312.

22 Andrews DL, Beames B, Summers MD \& Park WD. Characterization of the lipid acyl hydrolase activity of the major potato (Solanum tuberosum) tuber protein, patatin, by cloning and abundant expression in a baculovirus vector. Biochemical Journal 1988252 199-206.

Received 5 August 2008

Accepted 9 August 2008 